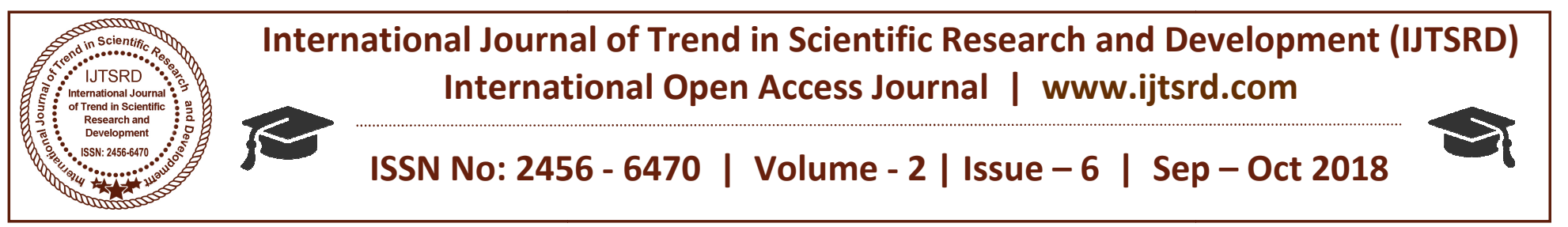

\title{
IOT Based Structural Health Monitoring
}

\author{
Junaid Rasool \\ B.Tech Student, Department of Electronics and Instrumentation Technology \\ University of Kashmir, Srinagar, Jammu and Kashmir, India
}

\begin{abstract}
Structural Health Monitoring is an emerging field of science and technology. The process of implementing a damage detection and characterization strategy for engineering structures is referred to as Structural Health Monitoring (SHM). The SHM process involves the observation of a system over time using periodically sampled dynamic response measurements from an array of sensors, the extraction of damagesensitive features from these measurements, and the statistical analysis of these features to determine the current state of system health. The research paper describes the piezo-vibrational sensor and accelerometer sensors to monitor the prototype of bridge.
\end{abstract}

Keyword: Sensor, SHM.

\section{INTRODUCTION}

regarding the integrity of the structure. First of all we should understand how the health of structure like buildings, bridges or any other civil structure is related and monitored by Electronics \& communication. The health of a structure is related and monitored in the similar fashion as the health of a human being is monitored by health monitoring devices and systems like Electronic blood pressure machine, Blood sugar checking device, Cardiovascular monitoring systems, ECGs, Activity monitoring systems etc. All these devices work by collecting the data from human body in the form of signals through electronic sensors and then converting into the electric signal. This data is stored processed and calibrated to any aspect of human health. This value is then compared to a set reference value and checked whether it is higher or lower or equal to it. Accordingly the health of a human body is monitored. Now in the similar fashion the health of a structure is monitored by Structural Health Monitoring Systems. characterization strategy for engineering structures is referred to as Structural Health Monitoring (SHM). Here damage is defined as changes to the material and/or geometric properties of a structural system, including changes to the boundary conditions and system connectivity, which adversely affect the system's performance. The SHM process involves the observation of a system over time using periodically sampled dynamic response measurements from an array of sensors, the extraction of damage-sensitive features from these measurements, and the statistical analysis of these features to determine the current state of system health. For long term SHM, the output of this process is periodically updated information regarding the ability of the structure to perform its intended function in light of the inevitable aging and degradation resulting from operational environments. After extreme events, such as earthquakes or blast loading, SHM is used for rapid condition screening and aims to provide real time, reliable information

\section{Problem Definition}

Bridges are defined as structures which provide a passage over a gap without blocking the passage beneath. It can be defined as the structure providing passage over an obstacle without closing the way beneath. The required passage can be over streams, road, a railway, pedestrian, or a canal. The paper focus on the technologies to be implemented to minimize the failures after construction.

\section{Objective}

The basic objective is to find the basic cause of the failure of the bridges. The study is conducted to help analyze why bridges fail. What signs should be observed when a bridge is about to fail and what possible actions must be taken to either prevent or to reduce the chances of the failure. Wireless sensor 
network technique is used to help the detection of the movement of the girders.

\section{Methodology}

The methodology starts with the investigation of the previous work and survey of research papers. My approach is based on microcontroller programming based on Arduino, which is an open source platform, thus making it more interactive and user friendly. With Arduino, we can build objects that can respond to and/or control light, sound, touch, and movement. Arduino has been used to create an amazing variety of things, including musical instruments, robots, light sculptures, games, interactive furniture, and even interactive clothing. The sensors used are PiezoVibration Sensor and Accelerometer (ADXL335). For online data logging Blynk app will be deployed. Blynk was designed for the Internet of Things. It can control hardware remotely, display sensor data, can store data, visualize it and do many other cool things.
There are three major components in the platform:

$>$ Blynk App - allows us to create amazing interfaces for your projects using various widgets we provide.

Blynk Server - responsible for all the communications between the Smartphone and hardware. We can use our Blynk Cloud or run private Blynk server locally. It is open-source, could easily handle thousands of devices and can even be launched on a Raspberry Pi.

Blynk Libraries - for all the popular hardware platforms - enable communication with the server and process all the incoming and out coming commands.

Every time we press a Button in the Blynk app, the message travels to the Blynk Cloud, where it magically finds its way to our hardware. It works the same in the opposite direction and everything happens in a Blynk of an eye.

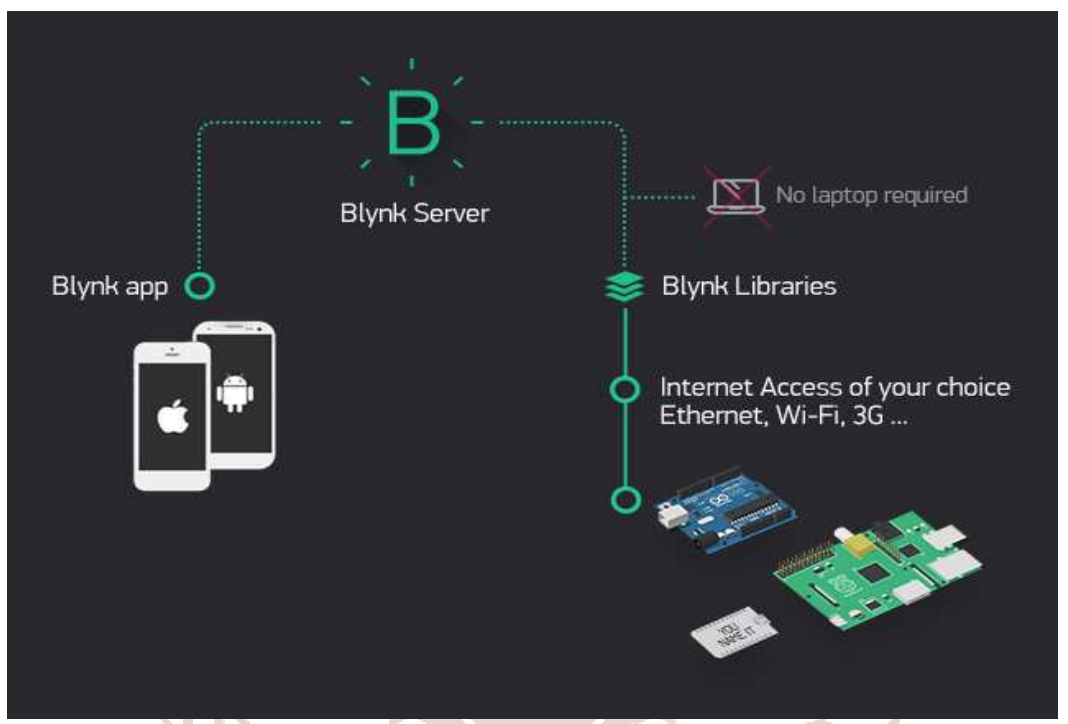

\section{Results}

Figure: Blynk Platform

The concept mainly consists of three parts Bridge prototype, Transmitter Module and Receiver Module.

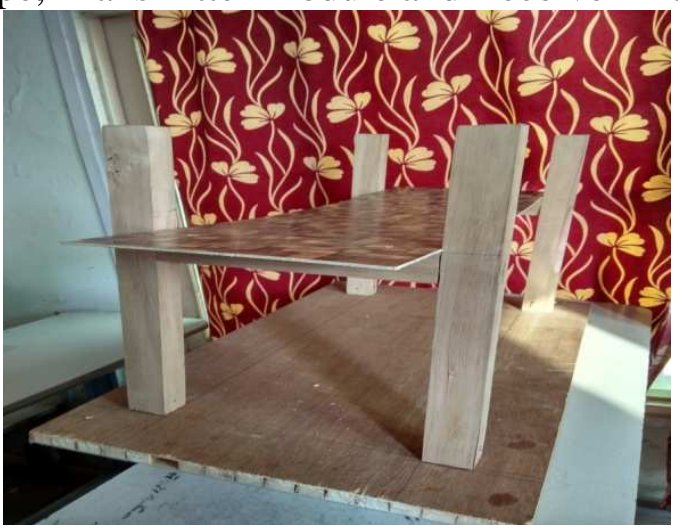

Figure: The bridge prototype

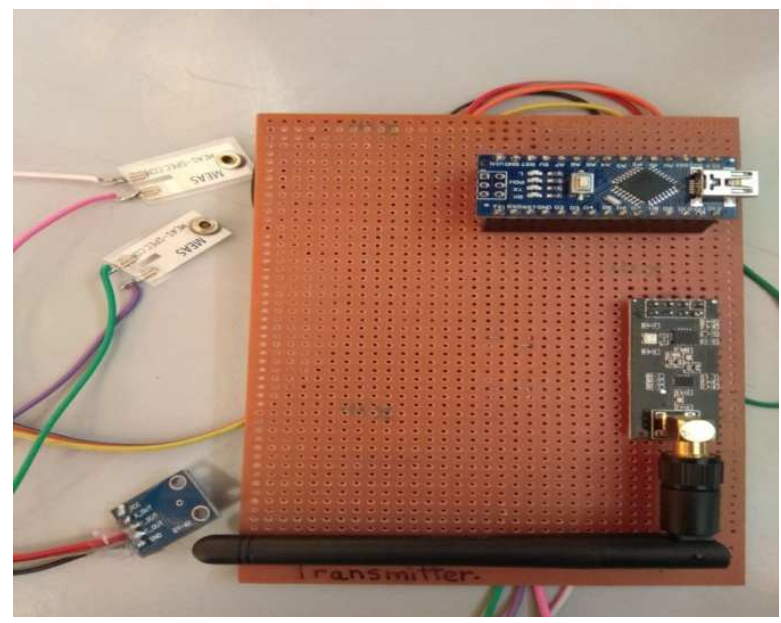

Figure: Circuit at the transmitter side 


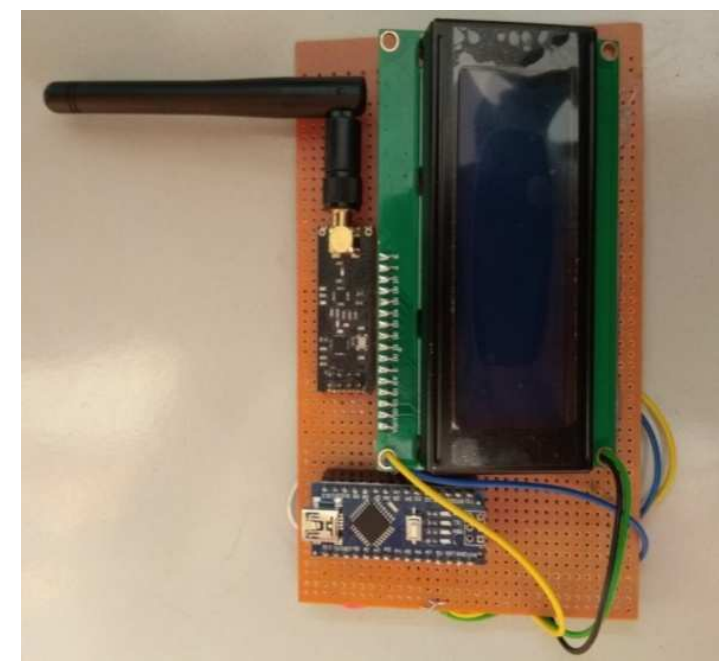

Figure: Circuit at the receiver side

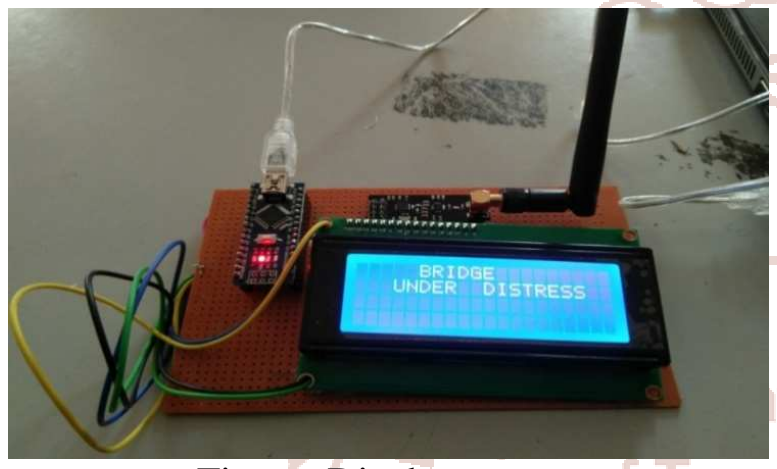

Figure: Display screen

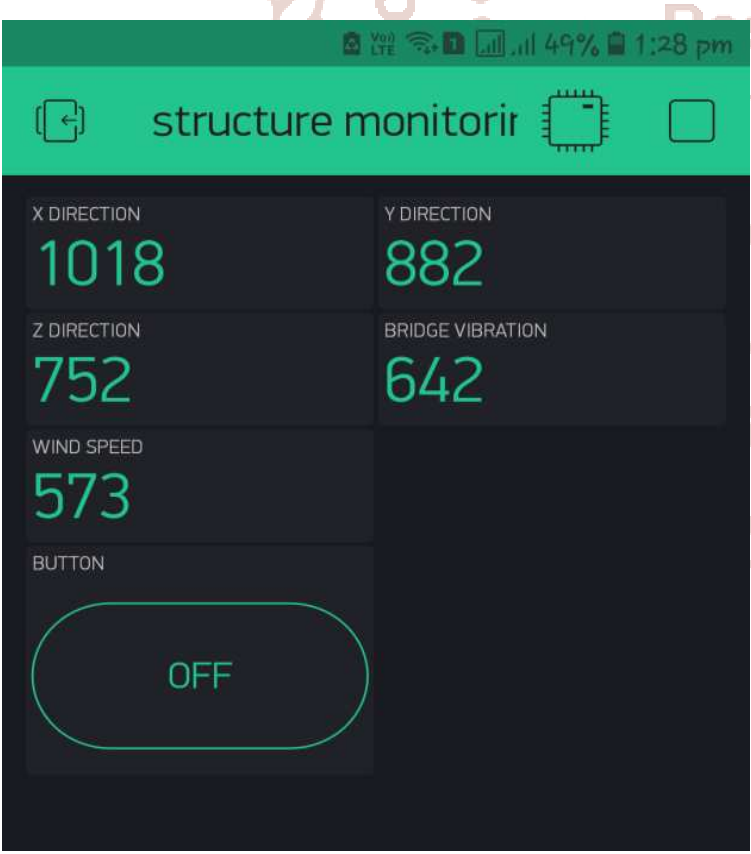

Figure: Structure monitoring on Blynk App

\section{Conclusion}

This paper was aimed at monitoring the health of a bridge so that adversaries can be averted. We can monitor other structures too. Knowing the health of the building we live in can be incredibly satisfying.
The fact of getting the information about various parameters of the health of a building on our mobile phone is very useful and handy. A weak structure is a threat to human life and property too. This paper provides the information about the various components used and the programming done using Arduino. Even structures have life and we (engineers) are here to protect it from various diseases.

\section{References:}

1. O. Yapar, P. K. Basu, P. Volgyesi, A. Ledeczi. Structural health monitoring of bridges with piezoelectric AE sensors. Elsevier, engineering failure analysis 2105.

2. Carlos Rodrigues, Carlos Felix, Armindo Lage, Joaquim Figueiras. Development of a long-term monitoring system based on FBG sensors applied to concrete bridges. Elsevier, engineering structures 2010.

3. A. M. AC. Bandara, R. M. S. U. P. Rathnayaka, W. A. I. P. Wijesooriya and P. B. R. Dissanayake. Sustainable solutions for retrofitting of bridges damaged by floods.

4. Standard specifications and code of practice for road bridges, section I (general features and design).

5. Standard specifications and code of practice for road bridges section II (loads and stresses).

6. D. Johnson Victor. Essentials of bridge engineering.

7. P. N Khanna. Indian practical civil engineers handbook.

8. Narayan, S. K. B and Basa, Ashok K. Foundation failure of bridges in Orissa :two Case studies.

9. Azmat Hussain, Saba Bashir, Saima Maqbool. Damage detection in bridges using image processing. International Journal of Civil Engineering and Technology (IJCIET).

10. R. Brannstrom, D. Granlund. Sensor monitoring of the bridge movement: system architecture. IEEE

11. Yong Bai, P. E, M. ASCE, William R. Burkett, P. E, and Phillip T. Nash, P.E. Rapid bridge replacement under emergency case situation .case study.

12. Azmat Hussain. Pile foundation for skewed bridge using slow and old machinery. IJSER 2105. 